\title{
Targeting of epidermal growth factor receptor (EGFR)-positive pancreatic cancer cell lines with cetuximab- conjugated near-infrared silver sulphide quantum dots
}

Peter L. Labib, Elnaz Yaghini, Mahshid Hashemkhani, Brian R. Davidson, Alexander J. MacRobert, et al.

Peter L. Labib, Elnaz Yaghini, Mahshid Hashemkhani, Brian R. Davidson, Alexander J. MacRobert, Marilena Loizidou, Havva Y. Acar, Stephen P. Pereira, "Targeting of epidermal growth factor receptor (EGFR)-positive pancreatic cancer cell lines with cetuximab-conjugated near-infrared silver sulphide quantum dots," Proc. SPIE 11070, 17th International Photodynamic Association World Congress, 1107013 (7 August 2019); doi:

$10.1117 / 12.2525086$

Event: 17th International Photodynamic Association World Congress, 2019, Cambridge, Massachusetts, United States 


\title{
Targeting of Epidermal Growth Factor Receptor (EGFR)-positive pancreatic cancer cell lines with cetuximab-conjugated near-infrared silver sulphide quantum dots
}

\author{
Peter L. Labib*a, Elnaz Yaghini ${ }^{\mathrm{b}}$, Mahshid Hashemkhanic ${ }^{\mathrm{c}}$, Brian R. Davidson ${ }^{\mathrm{b}}$, \\ Alexander J. MacRobert ${ }^{\mathrm{b}}$, Marilena Loizidou ${ }^{\mathrm{b}}$, Havva Y. Acar ${ }^{\mathrm{c}}$, Stephen P. Pereira ${ }^{\mathrm{a}}$ \\ ${ }^{a}$ Institute for Liver \& Digestive Health, University College London (Royal Free Campus), Royal \\ Free Hospital, Rowland Hill Street, London NW3 2PF, UK; ${ }^{b}$ Division of Surgery \& Interventional \\ Science, University College London (Royal Free Campus), Royal Free Hospital, Rowland Hill \\ Street, London NW3 2PF, UK; ' Department of Chemistry, Koç University, Rumelifeneri Yolu \\ 34450 Sariyer, Istanbul, Turkey
}

\begin{abstract}
Introduction: Fluorescence-guided surgery could potentially reduce local recurrence after pancreatic cancer resection. However, the ideal contrast agent for this purpose is not yet determined. The monoclonal antibody cetuximab targets the EGFR receptor, which is overexpressed in $64 \%$ of pancreatic cancers. We investigated the efficacy of near-infrared emitting silver sulphide Quantum Dot (QD)-cetuximab nanoconjugates for targeting EGFR-positive pancreatic cancer.

Methods: 2-Mercaptopropionic acid-coated QDs were prepared from $\mathrm{AgNO}_{3}$ and $\mathrm{Na}_{2} \mathrm{~S}$. Pancreatic cancer cell lines PANC-1 and CFPAC-1 were confirmed EGFR-positive using a commercial AlexaFluor488-cetuximab probe. Nonconjugated QD and cetuximab-conjugated QD (QD-cetuximab) toxicity was assessed after 24 and 48 hours using MTT assay. Fluorescence microscopy was performed following a) formaldehyde-fixed immunofluorescence and b) live staining with QD-cetuximab for four hours at concentrations corresponding to $0,10,50,100,200,400$ and $600 \mu \mathrm{g} \mathrm{ml}^{-1}$ of silver.

Results: Untargeted QDs were non-toxic in both cell lines after 48 hours at all investigated concentrations, whereas QDcetuximab was toxic at $100 \mu \mathrm{g} \mathrm{ml}^{-1}$ after 24 hours in PANC-1 and at $10 \mu \mathrm{g} \mathrm{ml}^{-1}$ in CFPAC-1. Fixed immunofluorescence demonstrated EGFR targeting by QD-cetuximab at concentrations of $50 \mu \mathrm{g} \mathrm{ml}^{-1}$ upwards in both cell lines. Live staining demonstrated similar efficacy of EGFR targeting up to $50 \mu \mathrm{g} \mathrm{ml}^{-1}$, although a reduction of fluorescence at higher concentrations was observed when compared to fixed immunofluorescence.
\end{abstract}

Conclusion: Silver sulphide QD-cetuximab nanoconjugates have the potential to target live EGFR-positive pancreatic cancer cells at doses of up to $50 \mu \mathrm{g} \mathrm{ml}^{-1}$. The reduction in QD fluorescence observed at higher concentrations is likely to be secondary to cetuximab toxicity.

Keywords: Quantum dots, epidermal growth factor receptor, pancreatic cancer, photodiagnostics, image-guided surgery

\section{INTRODUCTION}

\section{Pancreatic cancer}

Pancreatic cancer has been increasing in incidence over the last three decades and is now the $11^{\text {th }}$ most common cancer in the UK ${ }^{1}$. Unfortunately, due to aggressive tumor biology and the late onset of symptoms, most patients $(60 \%)$ are diagnosed with stage 3 or 4 disease ${ }^{2}$. As a result, only $10 \%$ of patients will undergo curative-intent surgery and only $28 \%$ will receive chemotherapy $\mathrm{y}^{2,3}$. Whilst complete surgical resection can cure the disease, recurrence is common with palliative treatments only providing a modest survival benefit ${ }^{4,5}$.

*peter.labib.16@ucl.ac.uk; phone +44 207433 2894; https://www.ucl.ac.uk/liver-and-digestive-health/

17th International Photodynamic Association World Congress, edited by Tayyaba Hasan, Proc. of SPIE

Vol. 11070, 1107013 - C 2019 SPIE · CCC code: 0277-786X/19/\$21 · doi: 10.1117/12.2525086 
Fluorescence-guided surgery

Fluorescence-guided surgery (FGS) is an emerging field that aims to assist the surgeon in the intraoperative identification of tumor to aid resection. Presently, surgeons rely on visual inspection and palpation of tissues to determine the location and extent of the tumor, with preoperative information usually provided by non-invasive imaging such as Computerized Tomography (CT) and Magnetic Resonance Imaging (MRI). Intraoperative imaging techniques (such as liver ultrasound to identify hepatic metastases) are only helpful at the beginning of the procedure as the tissue planes become distorted once resection begins. In addition, the rise of laparoscopic surgery has removed the tactile element of surgery, making the visual identification of residual tumor the most significant (and often only) intraoperative real time diagnostic tool. Research efforts have focused on finding ways to enhance the intraoperative visual detection of tumor to aid resection.

\section{Quantum dots}

Quantum dots (QDs) are nanometer-sized crystals of inorganic semiconductors with physical properties that are not present in the bulk form of the same material ${ }^{6,7}$. Some of their unique optical properties include ${ }^{8}$ :

a) High quantum yield (ratio of the number of photons absorbed to the number of photons emitted through fluorescence $)^{9}$;

b) Size and composition-tuneable light emission in the visible or near infrared (NIR) range;

c) A broad excitation window that allows a single light source to excite multiple QDs of varying emission bands at the same time (multiplexing) ${ }^{10}$; and

d) Chemical stability and photostability.

The typical structure of QDs for biomedical applications includes a core (with or without a shell) and a hydrophilic coating with or without a biomolecule of interest to functionalize the QD, such as antibodies specific to membrane-bound tumor biomarkers. These nanoconjugates can be used as contrast agents during FGS, potentially increasing the likelihood of complete tumor resection and reducing postoperative recurrence rates. Although human tissue is usually autofluorescent in the visible light range, it shows little NIR fluorescence. QDs emitting in the NIR I range (700-1000nm) show better promise as contrast agents in FGS as they allow for deeper tissue imaging whilst minimising interference from normal surrounding tissue.

\section{Silver sulphide QD-cetuximab nanoconjugates}

Our collaborators at the University of Koç developed a non-toxic silver sulphide $\mathrm{QD}^{11}$ coated with 2-mercaptopropionic acid (2-MPA) with or without cetuximab (Figure 1). Cetuximab is a monoclonal antibody targeting the human EGFR receptor, which is overexpressed in $64 \%$ of pancreatic cancers ${ }^{12}$. The nanoconjugates have a diameter of $\sim 12 \mathrm{~nm}$ and a peak emission at $800 \mathrm{~nm}$.

Aim

Our aim was to investigate the efficacy of NIR-emitting QDs and QD-cetuximab nanoconjugates in the targeting of EGFRpositive pancreatic cancer.

Figure 1 Diagrammatic representation of 2-MPA-coated silver sulphide quantum dots with or without cetuximab conjugation* QD-cetuximab

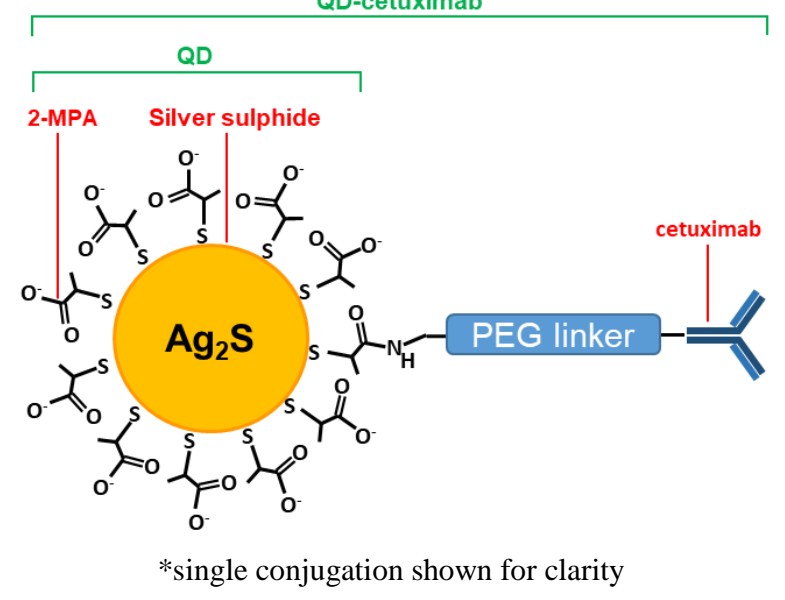




\section{METHODS}

\section{Cell culture}

PANC-1 was purchased from RIKEN BioResource Centre (RIKEN BRC, Tsukuba, Japan) and CFPAC-1 from ECACC (European Collection of Authenticated Cell Cultures, Salisbury, United Kingdom). PANC-1 was cultured in Dulbecco's modified Eagle's medium containing $4.5 \mathrm{~g} \mathrm{~L}^{-1}$ glucose and $0.58 \mathrm{~g} \mathrm{~L}^{-1} \mathrm{~L}$-glutamine (Lonza ${ }^{\circledR}$ ) supplemented with $10 \%$ Fetal

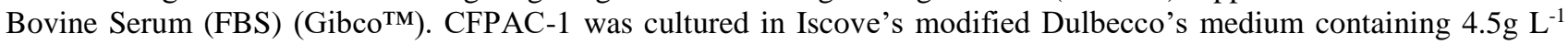
glucose, $4.0 \mathrm{mM}$ L-glutamine and HEPES buffer $\left(\mathrm{Gibco}^{\mathrm{TM}}\right)$ supplemented with $10 \%$ FBS.

\section{QD-cetuximab nanoconjugate synthesis}

2-MPA-coated silver sulphide QDs were synthesized from 2-MPA, $\mathrm{AgNO}_{3}$ and $\mathrm{Na}_{2} \mathrm{~S}$ using previously described methods ${ }^{11}$. Cetuximab was conjugated using thiolene chemistry and a heterofunctional PEG linker. Unbound cetuximab was removed by washing with Phosphate Buffered Saline (PBS) using a 100kDa centrifugal filter.

\section{Cell imaging}

PANC-1 and CFPAC-1 were confirmed EGFR-positive by fluorescence microscopy using a commercial AlexaFluor488cetuximab probe $\left(\mathrm{abcam}{ }^{\circledR}\right)$. To demonstrate that the synthesized QD-cetuximab nanoconjugates had a similar binding efficacy to the commercial probe, direct immunofluorescence of fixed cells was performed as follows: 24 hours postseeding, cells were washed three times in PBS and fixed using 4\% formaldehyde at room temperature. After 20 minutes, formaldehyde was removed, cells washed three times with PBS and PBS with 10\% FBS added (to prevent non-specific nanoconjugate binding). After 30 minutes, the PBS with 10\% FBS was aspirated and the cells then incubated at $37.0^{\circ} \mathrm{C}$ with increasing concentrations of QD-cetuximab (corresponding to $0,10,50,100,200,400$ and $600 \mu \mathrm{g} \mathrm{ml}^{-1}$ of silver) for one hour. Lastly, cells were washed three times with PBS and fluorescence microscopy performed using an Olympus ${ }^{\circledR}$ BX63 fluorescence microscope. To assess the EGFR targeting efficacy of QD-cetuximab in living cells, live targeting was performed as follows: 20 hours post-seeding, cells were washed three times in PBS and then incubated at $37.0^{\circ} \mathrm{C}$ with the aforementioned increasing concentrations of QD-cetuximab. After 4 hours, cells were washed three times with PBS, fixed with $4 \%$ formaldehyde at room temperature for 20 minutes and washed three times with PBS before having fluorescence microscopy performed as per fixed immunofluorescence.

\section{Evaluation of cytotoxicity}

The effect of QD and QD-cetuximab on cell metabolic activity was assessed using Methyl Thiazolyl Tetrazolium (MTT) assay. Cells were seeded on day 1, QD or QD-cetuximab in increasing concentrations $(0,10,50,100,200,400,600 \mu \mathrm{g}$ $\mathrm{ml}^{-1}$ ) were added either on day 2 (48 hour exposure) or day 3 (24 hour exposure) and MTT assay performed on day 4. Readings were taken using absorbance detection at $570 \mathrm{~nm}$ on an Infinite ${ }^{\circledR} 200$ PRO plate reader (Tecan®).

\section{Results}

\section{Fixed immunofluorescence studies}

The commercial probe confirmed overexpression of EGFR in both CFPAC-1 and PANC-1 (data not shown). Fixed immunofluorescence showed increasing NIR fluorescence with increasing doses of QD-cetuximab from 50 $\mu \mathrm{g} \mathrm{ml}^{-1}$ up to the maximum investigated dose of $600 \mu \mathrm{g} \mathrm{ml}^{-1}$, demonstrating good binding efficacy (Figure 2).

\section{Live immunofluorescence studies}

Whilst targeting efficacy was comparable until 50-100 $\mu \mathrm{g} \mathrm{ml}^{-1}$ in CFPAC-1 and 50 $\mu \mathrm{g} \mathrm{ml}^{-1}$ in PANC-1, higher doses resulted in a loss of near-infrared fluorescence (Figure 3).

\section{Metabolic activity studies}

Non-functionalized QDs (i.e. without cetuximab) showed no evidence of cellular toxicity after 24 or 48 hours incubation (one-way ANOVA 48 hours CFPAC-1 p=0.088, PANC-1 p=0.704). However, QD-cetuximab was shown to be toxic in CFPAC-1 at 24 and 48 hours from $10 \mu \mathrm{g} \mathrm{ml}^{-1}$ and in PANC-1 at 24 hours from $100 \mu \mathrm{g} \mathrm{ml}^{-1}$ and at 48 hours from $10 \mu \mathrm{g}$ $\mathrm{ml}^{-1}$ (one way ANOVA $\mathrm{p}<0.001$ for both cell lines at both time points). 
Figure 2 Fixed immunofluorescence of CFPAC-1 and PANC-1 using QD-cetuximab nanoconjugates*

$\mathrm{QD}$ concentration $\left(\mu \mathrm{g} \mathrm{ml}^{-1}\right.$ silver)

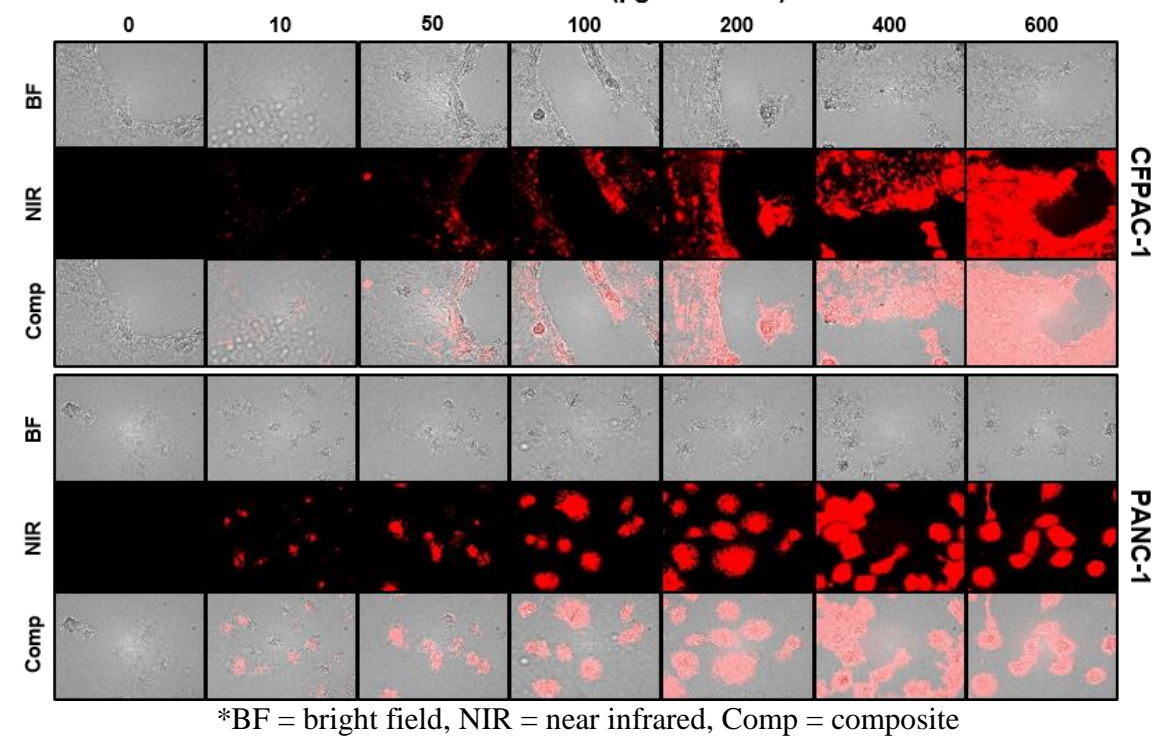

Figure 3 Live immunofluorescence of CFPAC-1 and PANC-1 using QD-cetuximab nanoconjugates*

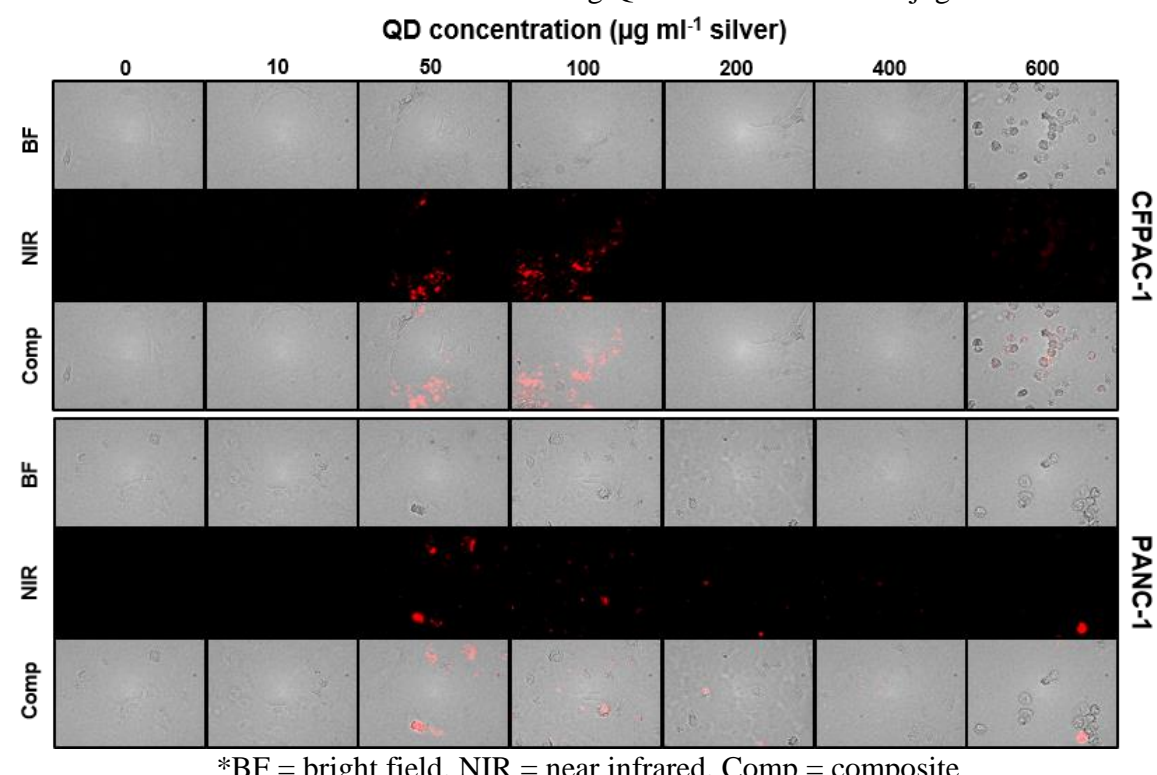

\section{Conclusion}

Non-functionalized QDs do not show evidence of cellular toxicity in two pancreatic cancer cell lines at doses of up to $600 \mu \mathrm{g} \mathrm{ml}^{-1}$. QD-cetuximab nanoconjugates have the potential to target EGFR-positive pancreatic cancer cells at doses of up to $50 \mu \mathrm{g} \mathrm{ml} \mathrm{m}^{-1}$, but show reduced targeting efficacy when compared to fixed immunofluorescence at the same concentrations. Given the MTT assay results, and the fact that cetuximab is known to cause antibody-dependent cellular cytotoxicity in a number of tumor cell lines ${ }^{13}$, the observed reduction in QD fluorescence at higher concentrations is likely to be secondary to cetuximab toxicity. For effective tissue-targeting in FGS using QD-antibody nanoconjugates, the optimal dose of antibody needs to be determined that allows for effective tumor targeting whilst minimising antibodyinduced cytotoxicity. Future research should also focus on discovery of membrane-bound biomarkers in pancreatic cancer that do not cause cellular toxicity when bound to as potential targets for functionalized quantum dots. 


\section{References}

[1] Cancer Research UK., "Pancreatic cancer mortality statistics" (2019).

[2] Pancreatic Cancer UK., "Pancreatic cancer statistics" (2019).

[3] Cancer Research UK., "Pancreatic cancer diagnosis and treatment statistics" (2019).

[4] Groot, V. P., Rezaee, N., Wu, W., Cameron, J. L., Fishman, E. K., Hruban, R. H., Weiss, M. J., Zheng, L., Wolfgang, C. L. and He, J., "Patterns, Timing, and Predictors of Recurrence Following Pancreatectomy for Pancreatic Ductal Adenocarcinoma:," Ann. Surg. 267(5), 936-945 (2018).

[5] Hidalgo, M., Cascinu, S., Kleeff, J., Labianca, R., Löhr, J.-M., Neoptolemos, J., Real, F. X., Van Laethem, J.-L. and Heinemann, V., "Addressing the challenges of pancreatic cancer: Future directions for improving outcomes," Pancreatology 15(1), 8-18 (2015).

[6] Auffan, M., Rose, J., Bottero, J.-Y., Lowry, G., Jolivet, J.-P. and Wiesner, M., "Towards a definition of inorganic nanoparticles from an environmental, health and safety perspective," Nat. Nanotechnol. 4, 634-641 (2009).

[7] Rosenthal, S., Chang, J., Kovtun, O., McBride, J. and Tomlinson, I., "Biocompatible quantum dots for biological applications," Chem. Biol. 18(1), 10-24 (2011).

[8] Matea, C., Mocan, T., Tabaran, F., Pop, T., Mosteanu, O., Puia, C., Iancu, C. and Mocan, L., "Quantum dots in imaging, drug delivery and sensor applications," Int. J. Nanomedicine 12, 5421-5431 (2017).

[9] Allen, M., "Measurement of fluorescence quantum yields," Technical note 52019, Therma Fisher Scientific, Wisconsin, USA (2010).

[10] Lidke, D. and Arndt-Jovin, D., "Imaging takes a quantum leap," Physiology 19(6), 322-325 (2004).

[11] Hocaoğlu, I., Çizmeciyan, M. N., Erdem, R., Ozen, C., Kurt, A., Sennaroglu, A. and Acar, H. Y., "Development of highly luminescent and cytocompatible near-IR-emitting aqueous Ag2S quantum dots," J. Mater. Chem. 22(29), 14674 (2012).

[12] Park, S. J., Gu, M. J., Lee, D. S., Yun, S. S., Kim, H. J. and Choi, J. H., "EGFR expression in pancreatic intraepithelial neoplasia and ductal adenocarcinoma,” Int. J. Clin. Exp. Pathol. 8(7), 8298-8304 (2015).

[13] Kimura, H., Sakai, K., Arao, T., Shimoyama, T., Tamura, T. and Nishio, K., "Antibody-dependent cellular cytotoxicity of cetuximab against tumor cells with wild-type or mutant epidermal growth factor receptor," Cancer Sci. 98(8), 1275-1280 (2007). 